\title{
The relativistic solar particle event of May 17th, 2012 observed on board the International Space Station
}

\author{
Francesco Berrilli ${ }^{1, *}$, Marco Casolino ${ }^{2,3}$, Dario Del Moro ${ }^{1}$, Luca Di Fino ${ }^{1,2}$, Marianna Larosa ${ }^{1,2}$, Livio Narici ${ }^{1,2}$, \\ Roberto Piazzesi ${ }^{1}$, Piergiorgio Picozza ${ }^{1,2}$, Stefano Scardigli ${ }^{1}$, Roberta Sparvoli ${ }^{1,2}$, Marco Stangalini ${ }^{4}$, \\ and Veronica Zaconte ${ }^{1,2}$ \\ 1 Department of Physics, University of Roma Tor Vergata, Via Ricerca Scientifica 1, 00133 Rome, Italy \\ ${ }^{*}$ Corresponding author: e-mail: berrilli@roma2.infn.it \\ 2 National Institute for Nuclear Physics, Tor Vergata Group, 00133 Rome, Italy \\ 3 RIKEN, Hirosawa, Wako, Saitama 351-0198, Japan \\ 4 INAF-Osservatorio Astronomico di Roma, 00040 Monte Porzio Catone (RM), Italy
}

Received 31 December 2013 / Accepted 1 May 2014

\begin{abstract}
High-energy charged particles represent a severe radiation risk for astronauts and spacecrafts and could damage ground critical infrastructures related to space services. Different natural sources are the origin of these particles, among them galactic cosmic rays, solar energetic particles and particles trapped in radiation belts. Solar particle events (SPE) consist in the emission of high-energy protons, alpha-particles, electrons and heavier particles from solar flares or shocks driven by solar plasma propagating through the corona and interplanetary space. Ground-level enhancements (GLE) are rare solar events in which particles are accelerated to near relativistic energies and affect space and ground-based infrastructures. During the current solar cycle 24 a single GLE event was recorded on May 17th, 2012 associated with an M5.1-class solar flare. The investigation of such a special class of solar events permits us to measure conditions in space critical to both scientific and operational research. This event, classified as GLE71, was detected on board the International Space Station (ISS) by the active particle detectors of the ALTEA (Anomalous Long Term Effects in Astronauts) experiment. The collected data permit us to study the radiation environment inside the ISS. In this work we present the first results of the analysis of data acquired by ALTEA detectors during GLE71 associated with an M5.1-class solar flare. We estimate the energy loss spectrum of the solar particles and evaluate the contribution to the total exposure of ISS astronauts to solar high-energy charged particles.
\end{abstract}

Key words. solar activity - flares - coronal mass ejection (CME) - energetic particle - ground-level enhancement (GLE) dose

\section{Introduction}

High-energy charged particles represent a severe radiation risk for astronauts and spacecrafts. Ground-based human technological infrastructures are not as substantially exposed to the effects of this kind of threats from space as space-based ones. Nevertheless they are often connected to services from space (GNSS, telecommunications, etc.) therefore they should be included in any debate on space radiation risks (RAENG 2013). Different natural sources originate these particles, among them galactic cosmic rays (GCR), solar energetic particles (SEP) and particles trapped in radiation belts. All these sources are affected by solar activity, both impulsive and due to the 11-year cycle.

In particular, solar particle events (SPEs) consist in the emission of high-energy Hydrogen and Helium nuclei (i.e., protons and alpha-particles, respectively), electrons and heavier particles from solar flares or shocks driven by solar plasma propagating through the corona and interplanetary space. When ground-based neutron monitors, which are detectors of the hadronic component in atmospheric secondary radiation related to collisions of primary cosmic rays with terrestrial atmospheric molecules, register abrupt increases in solar energetic particles (SEPs), we say that a ground-level enhancement (GLE) has occurred. Therefore, these events, typically in the GeV energy range, are the perfect example of Space Weather events where near relativistic and relativistic protons, propagating in the interplanetary magnetic field, directly connect physical processes occurring in the solar atmosphere with the Earth's surface.

The frequency and intensity of solar flares and SEP events increase during periods of maximum solar activity (e.g., Shea \& Smart 1990). Intense flares are less likely than faint ones. Indeed, power-law slopes are reported for peak fluxes, which are invariant through various solar cycles, using both observations and numerical models (e.g., Aschwanden \& Freeland 2012; Viticchié et al. 2006).

Although the association of GLE events with intense solar flares and coronal mass ejections (CMEs) is evident (e.g., Aschwanden 2012; Gopalswamy et al. 2012; Nitta et al. 2012), nevertheless GLEs have a sporadic occurrence and constitute a peculiar class of solar events in which particles are accelerated to relativistic energies (e.g., Aschwanden 2012). Only 71 GLE events have been reported in literature from the 1950s to the present (e.g., Papaioannou et al. 2013). Actually, as reported in Aschwanden (2012), a key question in the physics governing GLE events, which require acceleration processes able to produce energies $\gtrsim 1 \mathrm{GeV}$, is whether they originate from flares in the solar corona or from shocks 
driven by CMEs propagating through the corona and interplanetary space (e.g., Ellison \& Ramaty 1985). In his paper Aschwanden (2012) concludes that the sudden GLE component, present in virtually all GLEs, as reported by Vashenyuk et al. (2011), is consistent with a flare origin, while the delayed component can be generated either by acceleration and/or trapping in flare sites or by particles accelerated in coronal and interplanetary shocks.

During the current solar cycle 24 a single GLE event, associated with relativistic protons reaching the Earth, was recorded on May 17th, 2012. This event, classified as GLE71 (Firoz et al. 2014; Kudela 2013) and associated with an M5.1-class solar flare, was also detected on board the International Space Station (ISS) by the active particle detectors of the ALTEA (Anomalous Long Term Effects in Astronauts) experiment. This experiment was specifically designed to measure the effect of the exposure of crew members to space radiation and integrates several diagnostic technologies necessary to investigate the impacts that radiation has on the human central nervous system functions (Narici 2008). Therefore, it provides an unique assessment of the radiation environment inside the ISS. This task is accomplished by its active particle detector composed by silicon telescopes (either 3 or 6 ). The detector is able to achieve real-time measurements of the radiation environment with nuclear discrimination (Zaconte et al. 2008, 2010).

On board the ISS since July 2006, the ALTEA telescope was operative between August 2006 and November 2012 under different experimental setups (i.e., configuration, position and orientation) inside the USLab and Columbus modules of the ISS. The total effective observation time has been more than three years.

In this work we present the first results of the analysis of data acquired by ALTEA detectors during GLE71. The analysis of particle fluxes on board a spacecraft is particularly important because GLEs are able to produce various direct and indirect effects on circumterrestrial space and ground-based facilities. In particular, they can affect navigation and communication systems on board satellites, manned space missions or commercial aircraft operations (EURADOS WG5 2004). Although the contribution of GLEs to the total exposure of aircraft crews to high-energy charged particles is rather limited (Lantos \& Fuller 2003), nevertheless, GLEs increase the dose rate at typical aviation altitudes (Buetikofer \& Flueckiger 2011; Sihver et al. 2008).

\section{The May 17, 2012 M5.1-class solar flare}

The event of May 17 has been observed by various spacecraft detectors, such as the GOES satellites. The X-ray energy flux measured by the GOES Solar X-Ray Sensor in the broad energy band (100-800 pm) has been used to classify the May 17th, 2012 event. The X-ray energy flux peak reached $5.1 \times 10^{-5} \mathrm{~W} \mathrm{~m}^{2}$ representing an M5.1-class solar flare.

The source of the solar flare and associated CME was NOAA AR11476, located at the time at N11W76 (solar disk coordinates), on the western limb. The magnetogram of the region is shown in Figure 1. It was classified at the time of flaring as a Hale region $\beta / \beta$, that is, a double bipolar region. The same region, in the McIntosh classification, was identified as Cao/Fho. This indicates an active region composed of two sections: the first section of the spot is bipolar with at least one spot having a penumbra, it is open and asymmetric and there are few, if any, spots between leader and follower; the

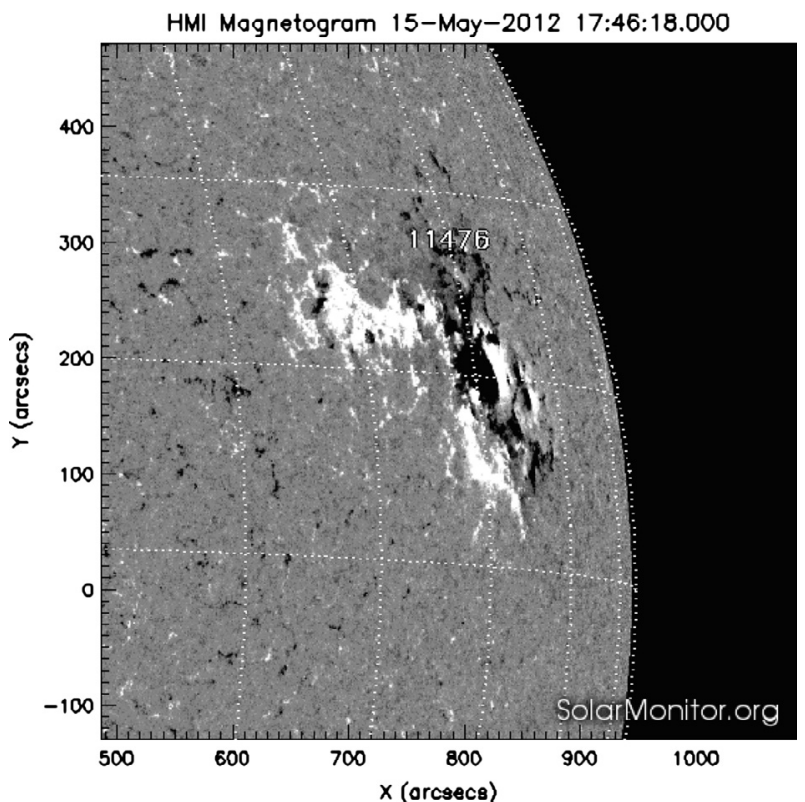

Fig. 1. SDO/HMI magnetogram of the active region NOAA AR11476, located at the time at N11W76 (solar disk coordinates). Data supplied courtesy of SolarMonitor.org.

second section is a bipolar sunspot group with penumbra on both ends, elongated in longitude, large, symmetric and open. The AR11476 region produced an M5.1 class flare at 01:47 UT (see Fig. 2, upper panel). More in detail, the flare started at 01:25 UT, peaked at 01:47 UT and stopped at 02:14 UT (see Fig. 2, lower panel), as reported at http://www.lmsal. com/solarsoft/last_events_20120517_1418/gev_20120517 0125. html.

The flare produced a halo CME which was recorded by the LASCO instrument aboard the SOHO spacecraft. CMEs that appear to completely surround the occulting disk of the observing coronagraphs in sky-plane projection are known as full-halo CMEs (e.g., Cid et al. 2012); CMEs that surround the disk with angles of more than $120^{\circ}$ are often referred to as halo CMEs. Halo CMEs are on average fast and wide and associated with flares of higher X-ray magnitude. The linear velocity of the associated CME was evaluated at $1582 \mathrm{~km} / \mathrm{s}$ (Papaioannou et al. 2013) with the LASCO instrument, while STEREO A and $B$ gave an estimate of $1302 \mathrm{~km} / \mathrm{s}$. The Wind/WAVES instrument recorded radio type III and II bursts, which is typical of solar flares. As reported in (Struminsky 2013) the HXR emission observed by ACS SPI was very low. Moreover, the author reports that proton fluxes measured by EPHIN/SOHO (25 MeV) and HET/STEREO (23.8-26.4 MeV) detectors showed the reservoir effect under quiet solar wind conditions, whereas a feeble proton enhancement was observed by STEREO-B. On the other hand, proton flux measured by the GOES 14 satellite registered a peak intensity of $150 \mathrm{pfu}$ (proton flux units: $1 \mathrm{pfu}=1$ particle $\left./\left(\mathrm{s} \mathrm{cm}^{2} \mathrm{sr}\right)\right)$. For a more detailed description of the May 17th, 2012 event we refer to Augusto et al. (2013), Firoz et al. (2014), Kudela (2013).

The Gamma-ray Burst Monitor (GBM) on board the FERMI satellite, sensible to X-rays and gamma rays with energies between $8 \mathrm{keV}$ and $40 \mathrm{MeV}$, shows a peak in NaI detector channels $6-12 \mathrm{keV}$ and $12-25 \mathrm{keV}$ simultaneous with the GOES maximum. At the Earth a geomagnetic index of $K p=4$ was measured (Augusto et al. 2013). The geomagnetic index $K p$ (planetary $K$-index) is a weighted average of $K$-index 

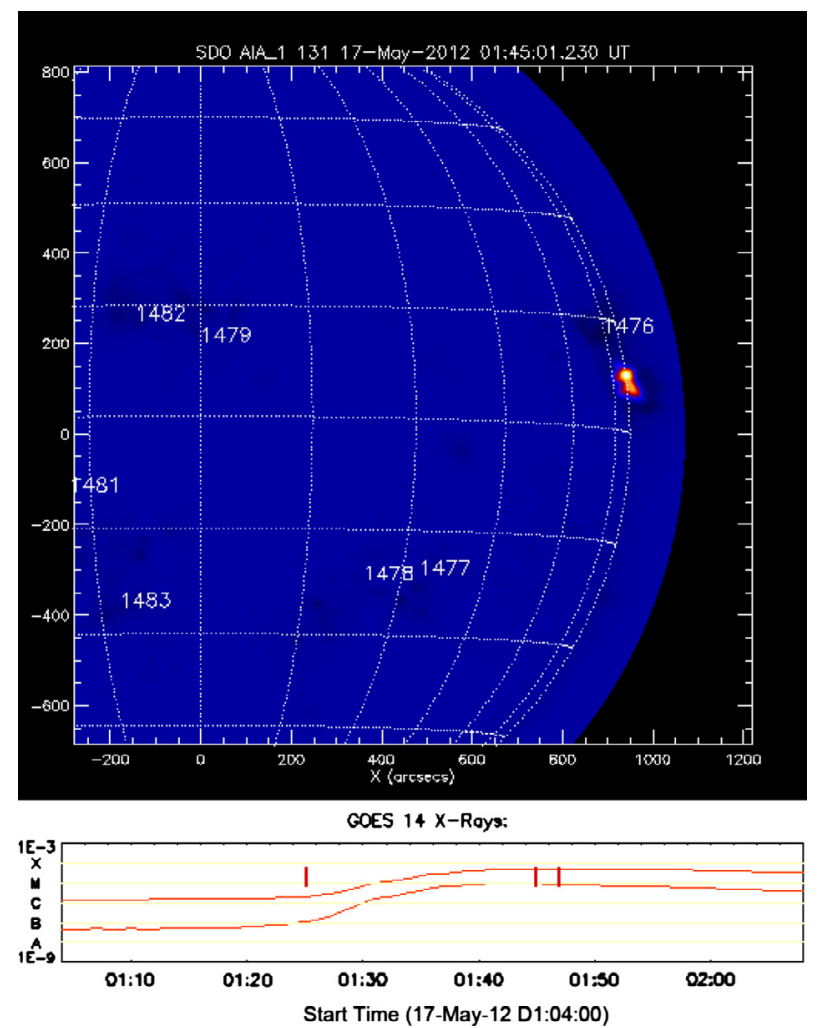

Fig. 2. Upper panel: Flare Locator Image showing AR11476 near west solar limb. Lower panel: GOES 14 X-Ray Flux measurement associated to the May 17th, 2012 SPE. Credits http://www.lmsal.com.

measurements at various observatories around the Earth. The $K$-index is an indicator of geomagnetic activity that measures disturbances in the horizontal component of earth's magnetic field and quantifies them with an integer ranging from 0 to 9 , with 1 being calm and 5 or more indicating a geomagnetic storm. It is based on the maximum fluctuations of horizontal components observed on a magnetometer during a 3-h interval.

A unique characteristic of this flare was observed through neutron monitor (NM) network measurements and IceTop Cherenkov detectors (Balabin et al. 2013; Mishev et al. 2014). Indeed, near 01:50 UT the NM worldwide network revealed the first enhancement caused by solar relativistic particles of cycle 24 and the first one since December 2006. Neutron monitors are ground-based detectors that measure cosmic rays, generally of energies above $500 \mathrm{MeV}$, through their interaction with the atmosphere and the production of secondary showers that include neutrons (Hatton 1971). As can be seen in Figure 3, the instruments that recorded the highest enhancements were those located at the South Pole, Oulu (Finland) and Apatity (Northern Russia), with moderate increases observed in other stations. The GLE71 was largely anisotropic (Mishev \& Usoskin 2013), indeed in the same plot we can see that the signal increases are not exactly organized according to rigidity cutoffs. The increase in particles measured by neutron monitors during the GLE is thought to be due to the configuration of the associated magnetic field orientation and strength, which does not block cosmic rays, but allows SEPs to be added to the total count. Finally, IceTop data analysis showed the increase in counting rate of individual IceTop discriminators averaged over $15 \mathrm{~min}$ from 02:05 to 02:20 UT. In this context The IceCube Collaboration, Aartsen et al. (2013), by using calculated and measured yield functions for the various detectors, has been able to estimate the energy spectrum of the solar particles and interpret the results in the perspective of the NM network.

\section{ALTEA-space particle detection on board the ISS}

The ALTEA-space particle detection system, called SDS (Silicon Detector System), includes six identical particle telescopes (SDU, Silicon Detector Units) arranged on an adaptable mounting system (Narici 2008; Di Fino et al. 2012). Each SDU is able to determine the energy loss and the trajectory of the penetrating cosmic rays. Each SDU consists of three pairs of silicon ladders, each ladder composed of two silicon chips segmented into 32 strips with $2.5 \mathrm{~mm}$ pitch. Strip segmentation on each ladder is alternatively oriented along orthogonal directions in order to use the two strip coordinates and the height of the ladder pair into the detector as three coordinates in space. Each silicon chip has a size of $8 \mathrm{~cm} \times 8 \mathrm{~cm}$ and a thickness of $380 \mu \mathrm{m}$. The geometrical factor of a single SDU is $230 \mathrm{~cm}^{2} \mathrm{sr}$. The energy resolution of the detector ranges from a threshold of $2.9 \pm 0.2 \mathrm{KeV} / \mu \mathrm{m}$ up to $800 \mathrm{KeV} / \mu \mathrm{m}$ (linear energy transfer [LET] in $\mathrm{Si}$ ), corresponding to ions between $\mathrm{Li}$ and $\mathrm{Fe}$ with a small energy interval for protons and He. The detector is triggered by pass-through particles that release more than the threshold on all the odd planes of an SDU. Further details are reported in Larosa et al. (2011) and in Di Fino et al. (2011).

The orbital information provided by the ISS motion permits the investigation of the angular distribution of particle directions. More in detail the three main geomagnetic zones (poles, equator and South Atlantic Anomaly) can be analyzed. ALTEA SDUs became operational in a 3D configuration for Survey (ALTEA-shield/Survey, part of an ESA sponsored experiment) from September 2010 until June 2012. In this experimental setup the SDUs were coupled to form three orthogonal silicon telescopes with 12 planes each (see Fig. 4).

In this work we used data acquired by the telescope SDU1. Indeed, SDU1 recorded the highest flux during the analyzed solar event, most likely due to a favourable coupling of detector orientation, lowly shielded position in the ISS and SEP directionality.

\section{Data analysis}

During the operation period of ALTEA Shield Survey (3D configuration), for about 2 years, the detectors of the experiment collected GCRs showing a typical North pole-South pole oscillation and periodical increments due to the passages over the South Atlantic Anomaly (SAA). Typically, the highest peaks correspond to passes through the SAA, where there is a large increase of the particle flux due to the contribution of the protons trapped in the inner radiation belt. Changes in this typical behaviour usually are the signature of a Solar Particle Event (SPE). In Figure 5 the collected particle flux, integrated over one minute, for the period May 8-23, 2012 is shown; on May 17 the flux increment is due to the SPE described in Section 2. The associated flux change is observed at 03:57 UT and during a few polar passages starting at about 09:50 UT (mainly South Pole passages). SEPs entered the geomagnetic shield during five South passages versus only one North passage (see five lower panel). Although the flux enhancement is clear when integrating the flux over a minute, its short duration and low intensity make the flux increase over the whole 


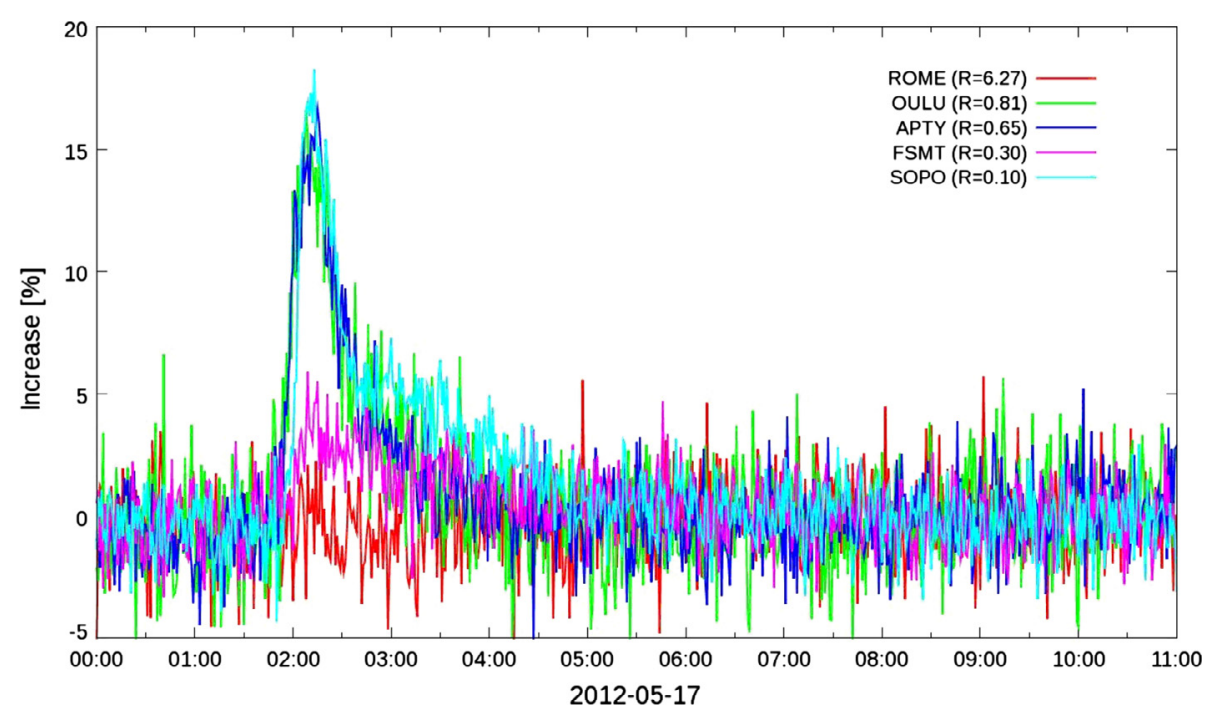

Fig. 3. The event of the May 17th, 2012 was associated with the only GLE, up to now, of cycle 24, and measurements from the global network of neutron monitors are shown. Different recorded enhancements from different stations are due both to the specific geomagnetic cutoff rigidities and to the anisotropy proper to the GLE 71. Data supplied courtesy of Neutron Monitor Data Base (NMDB) http://www.nmdb.eu/.

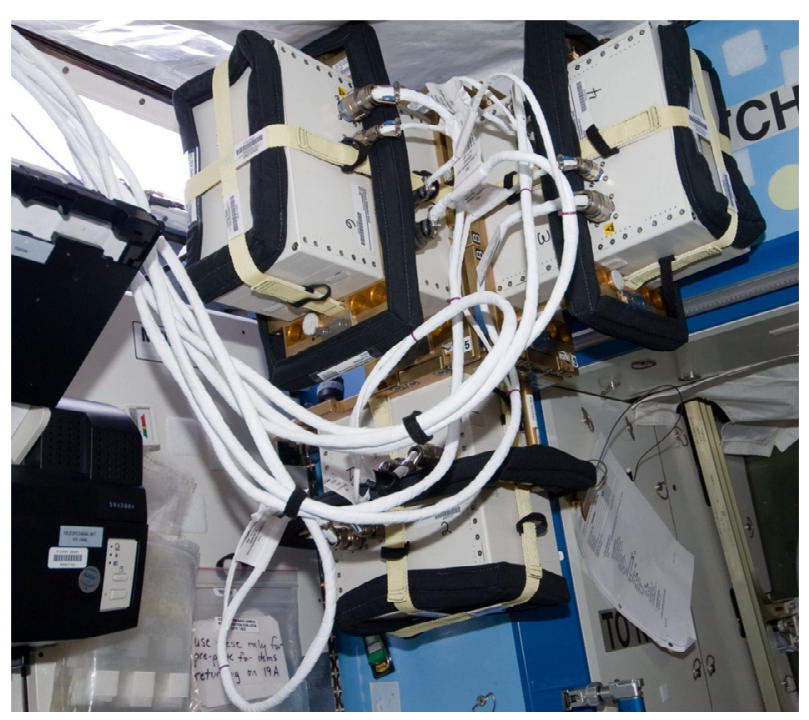

Fig. 4. ALTEA in position in the UsLab on board the ISS. The SDUs were coupled to form three orthogonal silicon telescopes with 12 planes each.

orbit hardly distinguishable when daily averaged (Fig. 6). When considering the daily average only over the polar regions (see below for a detailed definition) the effect is relevant as shown in Figure 7. The corresponding change in dose rates onboard ISS is reported in Figure 8.

The longitudes where solar particles are able to penetrate the geomagnetic shield causing the flux enhancement are shown in Figure 9 where the integrated particle flux measured at high latitudes (more than $+45^{\circ}$ and less than $-45^{\circ}$ ) is plotted versus longitude. The flux, for each day between the 15 th and 19 th of May, is integrated over $4^{\circ}$ longitude and normalized by the effective acquisition time and by the longitude bin. In Figure 9A, representing the northern passages, there is a weak flux increase due to the SPE only on May 17 th in a region centred at a longitude of about $-75^{\circ}$, while in the Southern passages (Fig. 9B) the SPE effect is more evident due to the lower geomagnetic cutoff.
As discussed in Section 3 ALTEA is able to measure the energy loss spectrum of detected particles. In Figure 10 the energy loss spectrum of the May 17th, 2012 SPE, integrated over the whole orbit for the entire day, is shown in comparison with a reference period of quiet sun (April 20-May 16). Both spectra are normalized by the effective time of detector acquisition, by the geometrical factor and by the energy loss unit. Due to the high statistics the quiet sun spectrum is less noisy than the one-day SPE event. Peaks corresponding to relativistic ions between Boron and Oxygen are clearly noticeable. Protons and Helium are detectable by ALTEA only in specific kinetic energy ranges, 25-45 MeV for protons and 25-250 MeV/ amu for Helium ions (Zaconte et al. 2008).

During the May 17th SPE a flux increase of $\sim 1.15$ is observable below $10 \mathrm{KeV} / \mu \mathrm{m}$, due to the proton component, while above this LET value (that is usually affected by the Helium component of the SPE) there is no significant flux enhancement. These results are supported by simulations performed with the SRIM2013 code (Ziegler 2004) which is able to calculate interaction of ions with matter using a Monte Carlo simulation based on the binary collision approximation with random selection of ion impact parameters. The SRIM2013 code calculated the maximum energy loss by protons triggering ALTEA to be $\sim 10 \mathrm{KeV} / \mu \mathrm{m}$ while the analogue simulations for Helium nuclei resulted in $35 \mathrm{KeV} / \mu \mathrm{m}$. Unfortunately, the poor statistics associated with the observed SPE event prevent us from investigating the energy loss spectrum beyond the LET value of $30 \mathrm{KeV} / \mu \mathrm{m}$.

As seen in Figure 9, the particles responsible for flux enhancement penetrate the geomagnetic shield mainly when ISS orbits pass through regions close to the magnetic poles. It is therefore interesting to analyze the variations of the spectrum integrated over the polar regions, where the energy cutoff is lower with respect to the equatorial region. This selection is performed with the constraint of McIlwain coordinate $L>3$. We recall that, given a point in space and the magnetic field line passing through it, $L$ is the distance (expressed in Earth radii) between the Earth's centre and the intersection between that field line and the equatorial plane. Therefore, the spectra integrated on $L>3$ select the particles penetrating the polar regions, where the energy cutoff is lower with respect to the 
F. Berrilli et al.: The Relativistic solar event of May 17th, 2012 on board the ISS
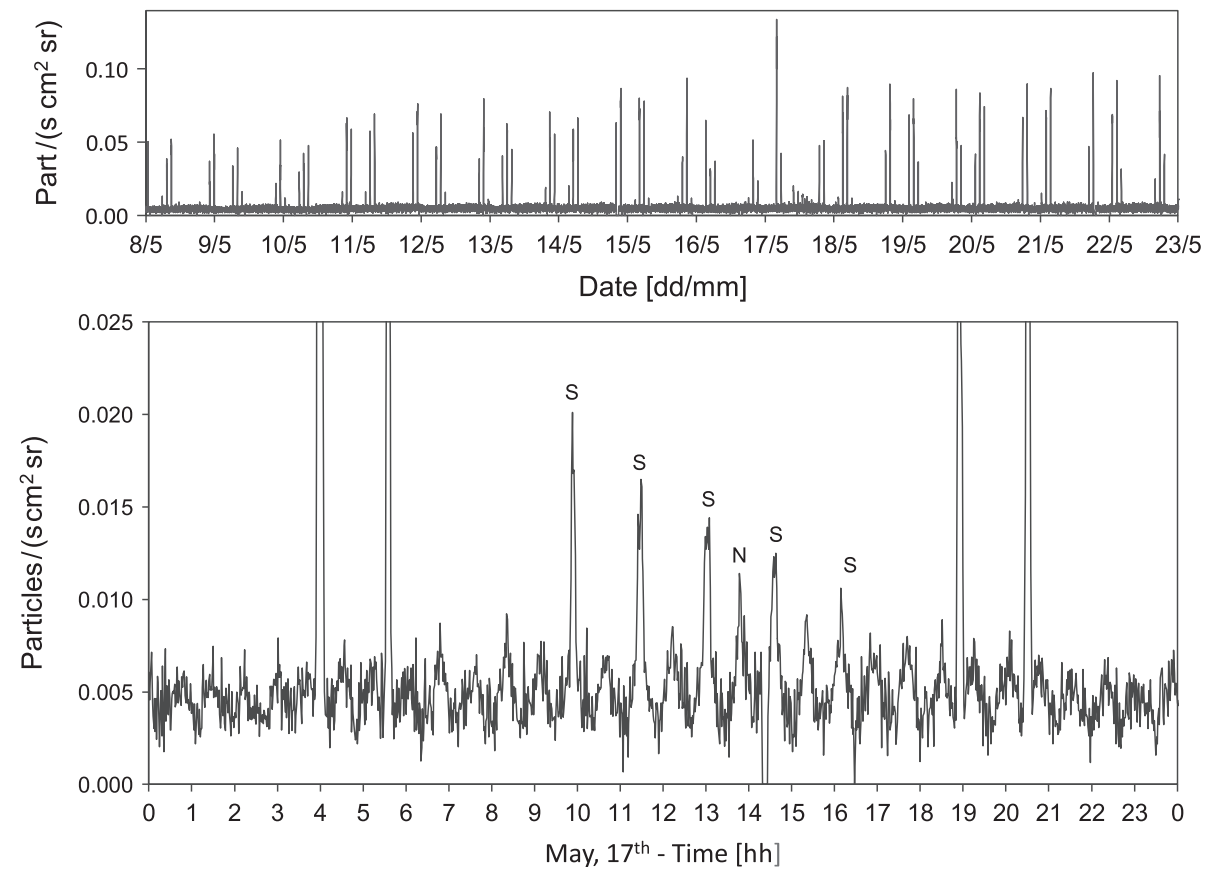

Fig. 5. Upper panel: ALTEA minute-averaged particle flux between the 8 th and 23 rd of May. All the typical particle flux modulations, due to the ISS passing into different geomagnetic cutoff regions, are shown: pole-equator 90-min oscillations, SAA passages (highest peaks) and longitude effect ( $24 \mathrm{~h}$ low frequency modulation due to orbit precession). Lower panel: Particle flux detail of May 17th, 2012 SPE flux increase over the poles: labelled $\mathrm{S}$ for south pole and $\mathrm{N}$ for north pole. Time on the $x$ axis is UT.

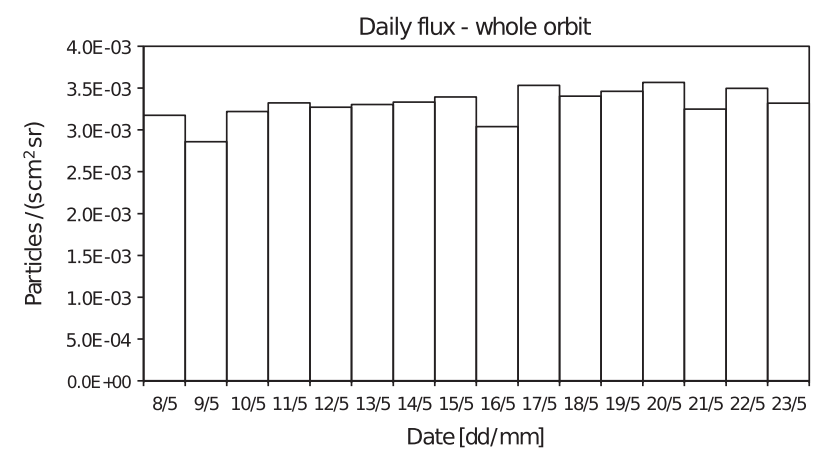

Fig. 6. ALTEA day-averaged flux between May 8 and 23: whole orbit passages.

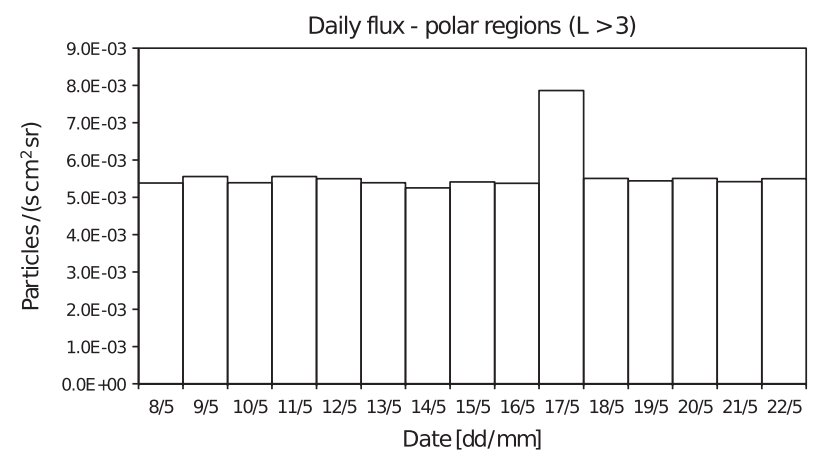

Fig. 7. ALTEA day-averaged flux between May 8 and 23: polar passages.

equatorial region. Comparing spectra reported in Figure 11 an overall increase of $\sim 1.5$ times the reference spectrum for energy loss less than $10 \mathrm{KeV} / \mu \mathrm{m}$ is observed, confirming that SPE flux

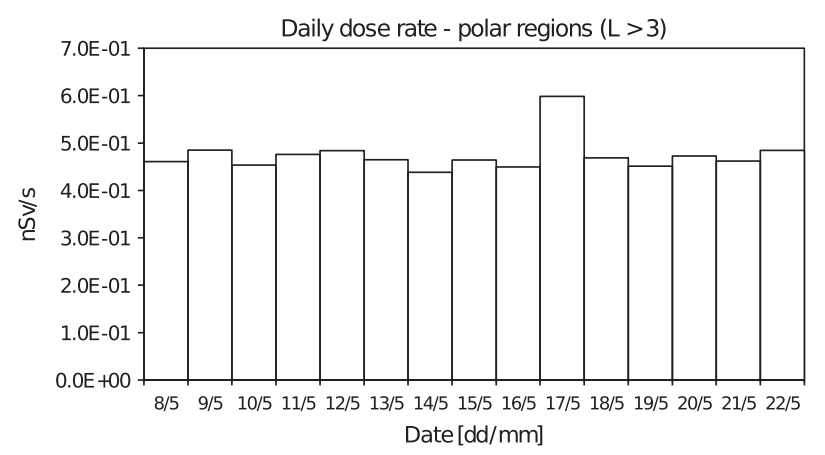

Fig 8. ALTEA day-averaged doses between May 8 and 23: polar passages.

enhancement is mainly caused by protons. It is worth noting that statistical errors computed for the SPE/reference spetrum ratio are below $10^{-2}$ for the components up to $10 \mathrm{KeV} / \mu \mathrm{m}$ confirming the statistical significance of the spectrum enhancement due to the SPE.

In order to answer the basic question of possible radiation risk for astronauts and spacecrafts due to high-energy charged particles (see Sect. 1) the analysis of ALTEA data is fundamental. More in detail, the evaluation of Dose Equivalent enhancement caused by the SPE is essential for assessing the correlated radiation risk. To calculate dose and Dose Equivalent, first we transform the LET in silicon into the LET in water according to the following formula from Benton et al. (2010):

$$
\log \left(\operatorname{LET}_{\mathrm{H}_{2} \mathrm{O}}\right)=-0.2902+1.025 \log \left(\mathrm{LET}_{\mathrm{Si}}\right) .
$$

The differential absorbed dose rate, in $(\mathrm{Gy} / \mathrm{s})$, is then obtained from:

$$
\text { Dose rate }=4 \pi \times 1.6 \times 10^{-9} \mathrm{LET}_{\mathrm{H}_{2} \mathrm{O}}
$$


J. Space Weather Space Clim. 4 (2014) A16

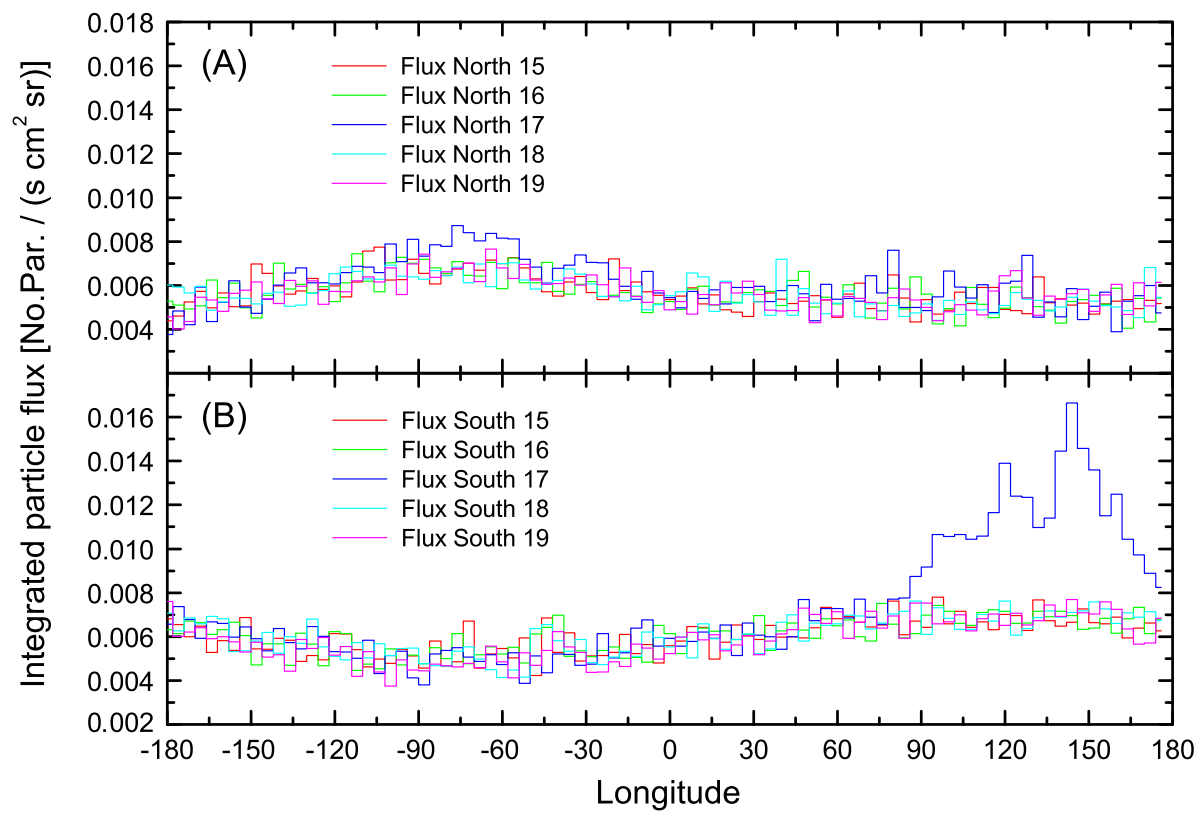

Fig. 9. Particle fluxes versus longitude integrated over latitude $>45^{\circ}$ (panel A) and $<-45^{\circ}$ (panel B) from 15th to 19th of May. Longitude bin size is $4^{\circ}$.

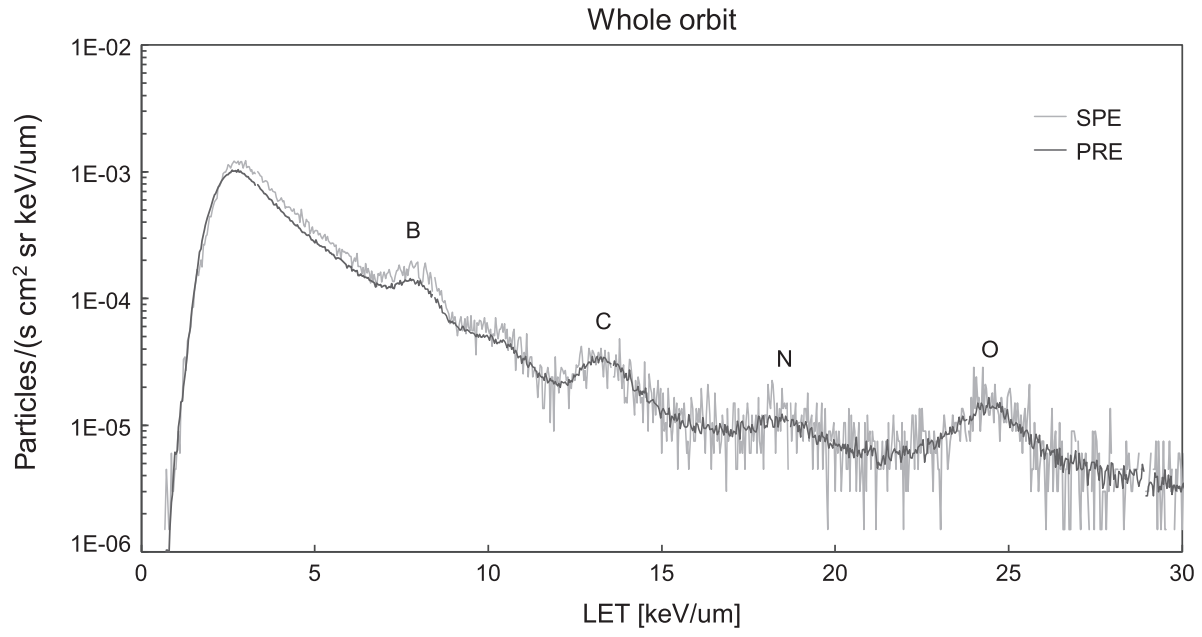

Fig. 10. The energy loss spectrum of the May 17th, 2012 SPE, integrated over the whole orbit, is shown in comparison with a reference period of quiet sun (April 20th-May 16th). Both spectra are normalized by the effective time of detector acquisition, by the geometrical factor and by the energy loss unit.

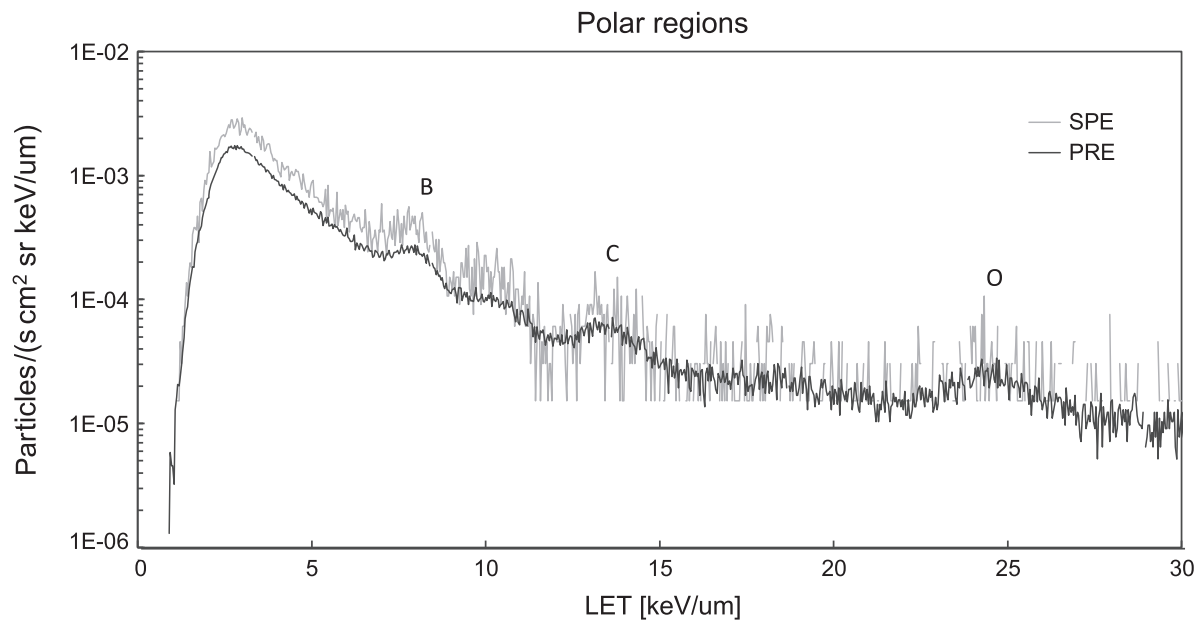

Fig. 11. The same as Figure 10 but using only polar passages. 


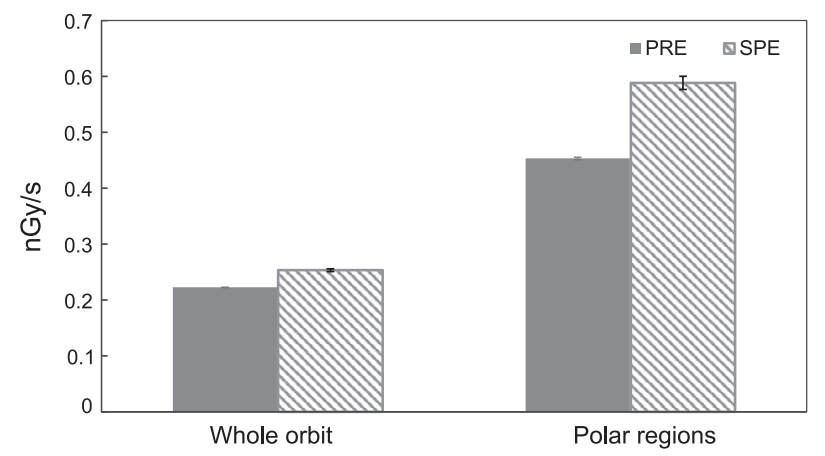

Fig. 12. Time-averaged dose rate during the SPE compared with the quiet sun period (PRE). The error bars represent statistical errors of 1-sigma.

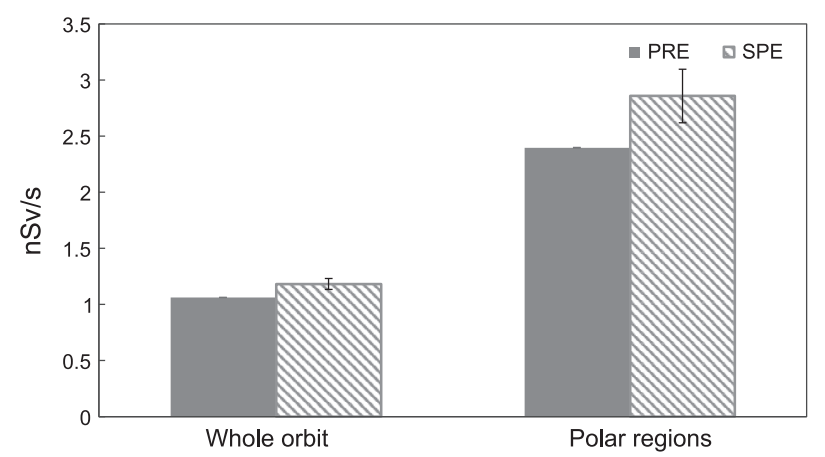

Fig. 13. Time-averaged Dose Equivalent rate during the SPE compared with the quiet sun period (PRE). The error bars represent statistical errors of 1-sigma

where the $\mathrm{LET}_{\mathrm{H}_{2} \mathrm{O}}$ rate is measured in $(\mathrm{KeV} / \mu \mathrm{m}) /\left(\mathrm{s} \mathrm{cm}^{2} \mathrm{sr}\right.$; Benton \& Benton 2001). The Dose Equivalent $H$ is calculated as $Q \times$ dose, where the quality factor $Q$ is defined as per ICRP recommendations (Mountford \& Temperton 1992). The Dose Equivalent rate is measured in Sv/s. From the analysis of the daily averaged dose rate plotted in Figure 12, we observe a light increase of the dose rate associated with the May 17th, 2012 SPE with respect to the dose measured during the preceding quiet sun period. As previously discussed, the increase is significant when only pole passages are considered.

The dose rate enhancement is more evident in Figure 12 and in Figure 13 where both dose and Dose Equivalent rates, computed for the observed SPE, are compared to the same quantities computed for a quiet sun period. All quantities are integrated over both the whole orbit and polar passages. The errors showed in the figure correspond to a 1-sigma statistical error. As noted above, the SPE dose increment is more evident in the polar regions (a factor $1.30 \pm 0.03$ at the poles compared with $1.13 \pm 0.04$ for the whole orbit). At the poles the Dose Equivalent variation $(1.2 \pm 0.1)$ is lower than the dose change due to the low quality factor of protons that constitute the main component of the SPE. To assess how SPEs affect doses in polar regions is important because of their similarity with outer space conditions.

\section{Conclusions}

In this work we report a first investigation about the effects produced on the ISS environment by the May 17, 2012 SEP event.
This event is important because it is associated with a GLE. This class of events, typically in the GeV energy range, represents the perfect example of Space Weather events where relativistic protons, propagating along the interplanetary magnetic field, directly connect physical processes occurring in the solar atmosphere with the Earth's surface. Moreover, the May 17 SPE is the only GLE event of solar cycle 24.

The ALTEA measurements provided a unique opportunity to assess the radiation risks for human permanence on board the ISS. From the analysis of daily averaged dose rates plotted in Figure 12 we observe a light increase of the dose rate associated with the May 17th SPE compared to the dose measured during the preceding quiet sun period. The dose variations of about $30 \%$ at the polar regions and $13 \%$ over the whole orbit are statistically relevant, while the correspondent changes in Dose Equivalent are barely significant. The May 17th SPE intensity as recorded by the ALTEA detector on board the ISS was, as measured, too low for a more detailed characterization of the SPE dynamics.

Although we confirm that the contribution of GLEs to the total exposure of astronauts, as of air crews, to high-energy charged particles is rather limited, nevertheless, GLEs do increase the dose rate during near polar passages.

A further opportunity offered by the ALTEA instrument is its capability to investigate the anisotropy of low-energy fluxes, while spanning the geomagnetic coordinates of its orbit. Indeed measurements above the Earth's atmosphere can give information about anisotropy below the atmospheric cutoff. Future analysis of ALTEA's data shall include an extended comparison with measures from the neutron monitor network, taking into account the different observed energetic ranges.

Acknowledgements. We thank Karel Kudela and the anonymous referee for their helpful comments on the manuscript. ALTEA has been funded by the Italian Space Agency (ASI) and the Italian National Institute for Nuclear Physics (INFN). The ALTEA program acknowledges support from ESA (experiment ALTEA shield) both on the ISS and on ground, NASA and the ISS crews for their support in orbit. The ISS is an international partnership with contributions from the United States, Russia, Japan and Canada, Europe. M. Larosa, R. Piazzesi and S. Scardigli acknowledge support by the SPARC: Space Awareness for Critical Infrastructure project. SPARC has been financed by the Prevention, Preparedness and Consequence Management of Terrorism and other Security-related Risks Programme - European Commission - Directorate-General Home Affairs under the Grant No.: HOME/2011/CIPS/AG/4000002119. Some images are courtesy of NASA/SDO and the AIA, EVE and HMI science teams. We acknowledge the NMDB database (www.nmdb.eu), founded under the European Union's FP7 programme (Contract No. 213007) for providing data. Data used was acquired at Rome, Oulu, Apatity, Fort Smith and South Pole (SOPO) stations. We acknowledge the SolarMonitor.org, http://www. Imsal.com, NOAA-NASA GOES, FERMI and RHESSI Projects for providing data. Part of the present analysis has been presented at the Tenth European Space Weather Week (ESWW10), November 18th until 22nd, 2013 at Antwerp in Belgium.

\section{References}

Augusto, C.R.A., V. Kopenkin, C.E. Navia, A.C.S. Felicio, F. Freire, et al.,Was the GLE on May 17, 2012 linked with the M5.1-class flare the first in the 24th solar cycle? [arXiv:1301.7055, astroph.SR], 2013.

Aschwanden, M.J., GeV particle acceleration in solar flares and Ground Level Enhancement (GLE) events, Space Sci. Rev., 171, 3-21, DOI: 10.1007/s11214-011-9865-x, 2012. 
Aschwanden, M.J., and S.L. Freeland, Automated solar flare statistics in soft X-rays over 37 years of GOES observations: The invariance of self-organized criticality during three solar cycles, Astrophys. J., 754, 112-126, DOI: 10.1088/0004-637X/754/2/112, 2012.

Balabin, Yu.V., E.V. Vashenyuk, B.B. Gvozdevsky, and A.V. GermanenkoPhysics of auroral phenomena, Proc. XXXVI Annual Seminar, Apatity, Kola Science Centre, Russian Academy of Science, pp. 103-105, 2013.

Benton, E.R., E.V. Benton, and A.L. Frank, Conversion between different forms of LET, Radiat. Meas., 45, 957-959, DOI: 10.1016/j.radmeas.2010.05.008, 2010.

Benton, E.R., and E.V. Benton, Space radiation dosimetry in lowEarth orbit and beyond, Nucl. Instrum. Methods Phys. Res., Sect. $B$, 184, 255-294, DOI: 10.1016/S0168-583X(01)00748-0, 2001.

Buetikofer, R., and E.O. Flueckiger, Radiation doses along selected flight profiles during two extreme solar cosmic ray events, Astrophys. Space Sci. Trans., 7, 105-109, DOI: 10.5194/astra-7-105-2011, 2011.

Cid, C., H. Cremades, A. Aran, C. Mandrini, B. Sanahuja, et al., Can a halo CME from the limb be geoeffective? J. Geophys. Res., 117, A11102, DOI: 10.1029/2012JA017536, 2012.

Di Fino, L., M. Casolino, C. De Santis, M. Larosa, C. La Tessa, L. Narici, P. Picozza, and V. Zaconte, Radiat. Res., 176, 397-406, DOI: 10.1667/RR2179.1, 2011.

Di Fino, L., V. Zaconte, A. Ciccotelli, M. Larosa, and L. Narici, Fast probabilistic particle identification algorithm using silicon strip detectors, Adv. Space Res., 50, 408-414, DOI: 10.1016/j.asr.2012.04.015, 2012.

Ellison, D.C., and R. Ramaty, Shock acceleration of electrons and ions in solar flares, Astrophys. J., 298, 400-408, DOI: 10.1086/163623, 1985

Firoz, K.A., W.Q. Gan, Y.P. Li, and J. Rodriguez-Pacheco, On the possible mechanism of the first ground level enhancement in cosmic ray intensity of solar cycle 24, Astrophys. Space Sci., 350 (1), 21-32, DOI: 10.1007/s10509-013-1729-2, 2014.

Gopalswamy, N., H. Xie, S. Yashiro, S. Akiyama, P. Mkel, and I. Usoskin, Properties of ground level enhancement events and the associated solar eruptions during solar cycle 23, Space Sci. Rev., 171, 23-60, DOI: 10.1007/s11214-012-9890-4, 2012.

Hatton, C., The neutron monitor, in Progress in elementary particle and cosmic ray Physics X, vol. 43, North Holland Publishing Co, Amsterdam, ISBN: 9780720414103, 1971.

Kudela, K., Space weather near Earth and energetic particles: selected results, J. Phys.: Conf. Ser., 409, article id. 012017, DOI: 10.1088/1742-6596/409/1/012017, 2013.

Lantos, P., and N. Fuller, History of the solar flare radiation doses on-board aircraft using a semi-empirical model and concorde measurements, Radiat. Prot. Dosim., 104, 199-210, 2003.

Larosa, M., F. Agostini, M. Casolino, C. De Santis, L. Di Fino, et al., On rates in the International Space Station during the December 2006 solar particle event, J. Phys. G: Nucl. Phys., 38, 095102, DOI: 10.1088/0954-3899/38/9/095102, 2011.

Mishev, A., and I. Usoskin, Analysis of recent GLEs with the newly computed NM yield function, 33rd International Cosmic Ray Conference, Rio de Janeiro, 2013.
Mishev, A.L., L.G. Kocharov, and I.G. Usoskin, Analysis of the ground level enhancement on May 17, 2012 using data from the global neutron monitor Network, J. Geophys. Res.: Space Phys., 119, 670-679, DOI: 10.1002/2013JA019253, 2014.

Mountford, P.J., and D.H. Temperton, Recommendations of the international commission on radiological protection, Eur. J. Nucl. Med., 19, 77-79, DOI: 10.1007/BF00184120, 1992.

Narici, L., Heavy ions light flashes and brain functions: recent observations at accelerators and in spaceflight, New J. Phys., 10, article id. 075010, DOI: 10.1088/1367-2630/10/7/075010, 2008.

Nitta, N.V., Y. Liu, M.L. DeRosa, and R.W. Nightingale, What are special about ground-level events? Space Sci. Rev., 171, 61-83, DOI: 10.1007/s11214-012-9877-1, 2012.

Papaioannou, A., G. Souvatzoglou, P. Paschalis, M. Gerontidou, and H. Mavromichalaki, The first ground-level enhancement of solar cycle 24 on 17 May 2012 and its real-time detection, Solar Phys., 289, 423-436, DOI: 10.1007/s11207-013-0336-2, 2013.

EURADOS, Cosmic Radiation Exposure of Aircraft Crew, Office for Official Publications of the European Communities, Luxembourg, ISBN: 92-894-8448-9, 2004.

RAENG, Extreme space weather: impacts on engineered systems and infrastructure, Royal Academy of Engineering, London, ISBN: 1-903496-95-0, 2013.

Shea, M.A., and D.F. Smart, A summary of major solar proton events, Solar Phys., 409, article id. 012148, DOI: $10.1007 / \mathrm{BF} 00152170,1990$.

Sihver, L., D. Matthiae, T. Koi, and D. Mancusi, Dose calculations at high altitudes and in deep space with GEANT4 using BIC and JQMD models for nucleusnucleus reactions, New J. Phys., 10, article id 105019, DOI: 10.1088/1367-2630/10/10/105019, 2008

Struminsky, A., Large SEP events of 2012: proton onset and source function, J. Phys.: Conf. Ser., 409, article id. 012148, DOI: 10.1088/1742-6596/409/1/012148, 2013.

The IceCube Collaboration, M.G. Aartsen, R. Abbasi, Y. Abdou, M. Ackermann, J. Adams, et al., Ground level enhancement of May 17, 2012 observed at South Pole (paper 0368) [arXiv:1309. 7006v2, astro-ph.HE], 2013.

Vashenyuk, E.V., Yu.V. Balabin, and B.B. Gvozdevsky, Features of relativistic solar proton spectra derived from ground level enhancement events (GLE) modeling, 2011, Astrophys. Space Sci. Trans., 7, 459-463, DOI: 10.5194/astra-7-459-2011, 2011.

Viticchié, B., D. Del Moro, and F. Berrilli, Statistical Properties of Synthetic Nanoflares, Astrophys. J., 652, 1734-1739, DOI: 10.1086/508332, 2006.

Zaconte, V., F. Belli, V. Bidoli, M. Casoline, L. Di Fino, et al., ALTEA: The instrument calibration, 2008, Nucl. Instrum. Methods Phys. Res. B, 266, 2070-2078, DOI: $10.1016 /$ j.nimb.2008.02.072, 2008.

Zaconte, V., M. Casolino, L. Di Fino, C. La Tessa, M. Larosa, et al., High energy radiation fluences in the ISS-USLab: Ion discrimination and particle abundances, Radiat. Meas., 45, 168-172, DOI: 10.1016/j.radmeas.2010.01.020, 2010.

Ziegler, J.F., SRIM-2003, Nucl. Instrum. Methods Phys. Res., Sect. $B$, 219, 1027-1036, DOI: 10.1016/j.nimb.2004.01.208, 2004

Cite this article as: Berrilli F, Casolino M, Del Moro D, Di Fino L, Larosa M, et al.: The relativistic solar particle event of May 17th, 2012 observed on board the International Space Station. J. Space Weather Space Clim., 2014, 4, A16. 